\title{
Design of Tapered Flatted End Tube for Tension and Compression Loading
}

\author{
Pravin S.Patil, I. P. Sonar
}

\begin{abstract}
Hollow circular tube has the most efficient section in space structure. Hollow circular tube joint either welding or bolting but welding aluminum structural alloys lead to unavoidable annealing which can reduce efficiency to the order of only $50 \%$. The efficiency of riveted and bolted joints is usually assumed to be around $75 \%$ in both aluminum and steel construction. Welded connections give the maximum strength in steel space structures and used for large spans. Hollow circular tube flatted at the end for single bolted connection and at flatted end increase this thickness for reducing stresses and this thicker plate make a hole for single bolted connection. Study this performance for varies the tapered flattened angle tube. Apply vertically upward and downward incremental load on the tapered flatted end tube at fixed bolted joint in ANSYS. Run this model for maximum shear stress and maximum principal stress. Find out the allowable tensile and compression stress in simple hollow circular tube in SAP2000 as per IS800-2007.. Find out the design load as per maximum shear stress theories and maximum principal stress theories.
\end{abstract}

Keywords: Tapered flatted end tube, theories of failure, Design load (Capacity).

\section{INTRODUCTION}

When some external load is applied on the body, the stresses and strains are produced in the body. The stresses are directly proportional to the strains within the elastic limit. This means when load is removed, the body will be return to its original shape. There is no permanent deformation in the body. (2) However, if stresses produced in the body due to the application of the load, is the beyond the elastic limit, the permanent deformation occur in the body, the body is said to have "failed". This should be clear that failure does not mean rupture of the body. (3)Let us consider the failure (or Permanent deformation) of a bar in a simple tensile test. The tensile stress is proportional to the tensile strain up to elastic limit. This means that there is definite value of tensile stress up to elastic limit. Beyond the elastic limit if the tensile stress increases, the failure of bar will takes place. At this stage, why the failure take place? It may be due to the increase of tensile stress, and anyone of these may be deciding factor of the failure of the bar.

Revised Manuscript Received on August 30, 2019.

* Correspondence Author

Pravin S.Patil*, Lecture, Department of Civil Engineering, College of Engineering, Pune (Maharashtra), India.

I. P. Sonar, Department of Civil Engineering, College of Engineering, Pune (Maharashtra), India.

(C) The Authors. Published by Blue Eyes Intelligence Engineering and Sciences Publication (BEIESP). This is an open access article under the CC BY-NC-ND license (http://creativecommons.org/licenses/by-nc-nd/4.0/)
Certain theories, the failure takes place when a certain limiting value is reached by one of following.

1. The maximum principal stresses.

2. The maximum principal strain

3. The maximum strain energy

4. The maximum shear strain energy

5. The maximum strain energy

$\sigma 1, \sigma 2, \sigma 3=$ principal stresses in any complex system $\sigma^{*}=$ tensile or compressive stress at the elastic limit.

Maximum Principal Stress theory

According to this theory, the failure of a material will be take place when the maximum principal tensile stress $(\sigma 1)$ in the complex system should be equal to or greater than of the maximum stress at the elastic limit in simple tension or the minimum principal stress reaches the value of the maximum stress at elastic limit in simple compression.

Let in a complex three dimensional stress system,

$\sigma 1, \sigma 2, \sigma 3=$ Principal stresses at a point in three perpendicular directions. The stresses $\sigma 1$ and $\sigma 2$ are tensile and $\sigma 3$ is compressive. Also $\sigma 1$ is more than $\sigma 2$. $\sigma^{*} \mathrm{t}=$ tensile stress at elastic limit in simple tension $\sigma^{*} \mathrm{c}=$ compressive stress at elastic limit in simple compression.

Then according this theory, the failure will take place if $\sigma 1 \geq \sigma^{*} \mathrm{t}$ in simple tension

$|\sigma 3| \geq \sigma^{*} \mathrm{c}$ in simple compression

This is simplest and oldest theory of failure and is known as

Rankine's theory. If the maximum principal stress is the design criterion, then maximum principal stress must not exceed the permissible stress $(\sigma \mathrm{t})$ for the given material.

Hence $\sigma 1=\sigma \mathrm{t}$

Where $\sigma \mathrm{t}=$ permissible stress and is given by

$\sigma \mathrm{t}=\sigma^{*} \mathrm{t} /$ safety factor

\section{Maximum shear stress theory}

This theory is due to Guest and Tresca and therefore known as Guest's theory. According to this theory, the failure of a material will occur when the maximum shear stress in a material reaches the value of maximum shear stress in simple tension at the elastic limit. The maximum shear stress in the material is equal to half the difference between maximum and minimum principal stress.

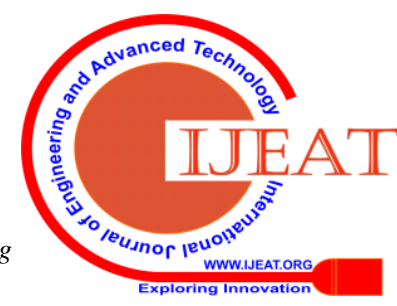


If $\sigma 1, \sigma 2$ and $\sigma 3$ are principal stresses at a point in a material for which $\sigma^{*} \mathrm{t}$ is the principal stress in simple tension at elastic limit, thenMaximum shear stress in the material $=$ half of difference of maximum and

minimum principal stresses.

$$
=1 / 2(\sigma 1-\sigma 3)
$$

In case of simple tension, at the elastic limit the principal stresses are $\sigma^{*} \mathrm{t} 0,0$

Maximum shear stress in simple tension at elastic limit.

$=$ Half of difference of maximum and minimum principal stresses

$=1 / 2 \sigma^{*} \mathrm{t}$

For failure material

$$
\begin{gathered}
1 / 2(\sigma 1-\sigma 3) \geq 1 / 2 \sigma^{*} \mathrm{t} \\
\text { Or } \\
(\sigma 1-\sigma 3) \geq \sigma^{*} \mathrm{t}
\end{gathered}
$$

For design the following equation should be used

$$
1 / 2(\sigma 1-\sigma 3)=1 / 2 \sigma \mathrm{t}
$$

$\sigma \mathrm{t} \quad=$ Allowable simple tensile stress (3)

\section{Analysis Setup:}

Modeled tapered flatted end tube and analysis setup details are furnished below

Length of Member - 630mm

Member- CHS (Circular Hollow section)

Member Size - CHS 50 mm OD, 2mm thick with flatted end Material - Fe250

Boundary Condition -bottom end fixed and top end pinned Loading- $10 \mathrm{KN}$ to $50 \mathrm{KN}$.

However a SAP analysis is made for simple hollow circular the tube. This is performed to check the member forces and deflection theoretically.

\section{Analysis in SAP 2000:}

Analysis of hollow circular tube is made in SAP 2000.For all the lines; geometry of hollow section with size $50 \mathrm{~mm}$ outer diameters and $2 \mathrm{~mm}$ thickness is assigned. Material steel with yield strength $250 \mathrm{MPa}$ (Fe250) is assigned to the tube. Bottom end is fixed, while the tube is loaded to incremental load. To find out design load for given section of tube in SAP2000 as per IS $800-2007$ as shown in fig 1.

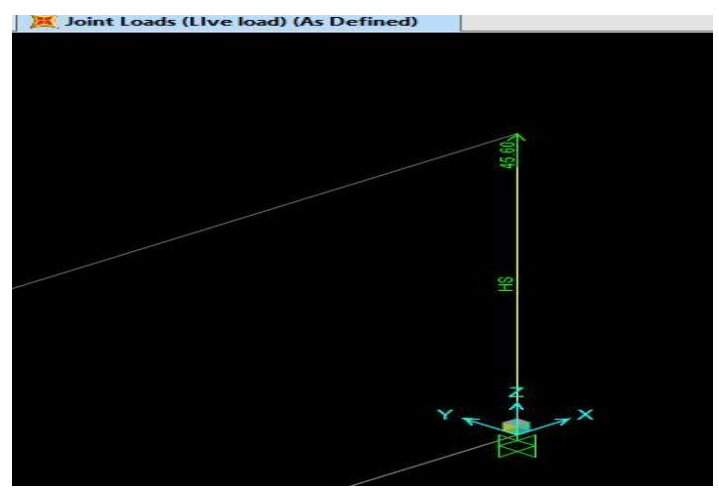

Fig 1. Hollow Circular Tube
Allowable tensile stress and compression stress are calculated in SAP2000 as per IS800- 2007 Allowable tensile stress at elastic limit in simple tension $=151.197 \mathrm{Mpa}$ Allowable compression stress at elastic limit in simple compression $(\sigma \mathrm{c})=140.255 \mathrm{Mpa}$

\section{Tapered flattened end tube-}

Hollow circular tube tapered flatted at the end for single bolted connection as shown in fig. The geometric details of tapered flatted end given in table 1 and fig 2 . After flattened end tube the maximum stresses have occurred at the end so for reducing this stresses increase flattened end thickness and after increasing this thickness it should be known as tapered flattened end tube. The tapered flattened end tube has more effective as compare to flattened end tube. Now in tapered flattened end tube the interested area should angle of tapered flattened end tube. In this study we have changing angle of tapered flattened end tube and analyze the stress calculation on ANSYS software. Create this model on Catia and import in workbench. While changing this angles ' $x$ ' values should varying as shown in table. The angles of tapered flattened end tube are $5^{\circ}, 10^{\circ}, 15^{\circ}, 20^{\circ}, 30^{\circ}, 45^{\circ}$ and $50^{\circ}$. Study this performance on ANSYS

$\mathrm{L}$

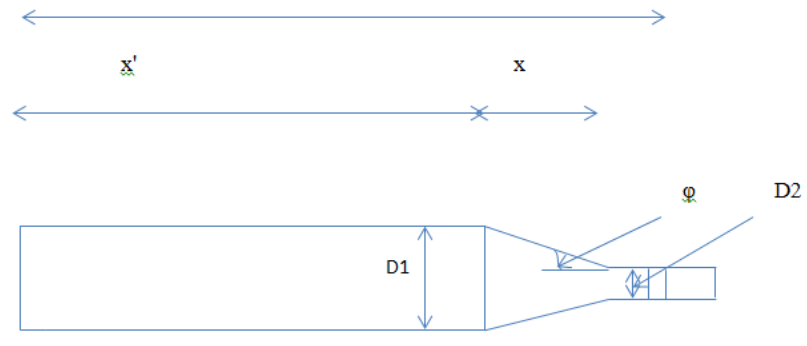

Fig 2. Modeling details

Table 1) Modeling Elements for different models

\begin{tabular}{|c|c|c|c|c|c|}
\hline $\begin{array}{c}\text { Angle } \\
(\varphi)\end{array}$ & $\mathrm{D} 1$ & $\mathrm{D} 2$ & $\mathrm{x}^{\prime}$ & $\mathrm{x}$ & $\mathrm{L}$ \\
\hline $5^{\circ}$ & 50 & 10 & 401.399 & 228.601 & 630 \\
\hline $10^{\circ}$ & 50 & 10 & 516.575 & 113.425 & 630 \\
\hline $15^{\circ}$ & 50 & 10 & 555.359 & 74.641 & 630 \\
\hline $20^{\circ}$ & 50 & 10 & 575.359 & 54.641 & 630 \\
\hline $30^{\circ}$ & 50 & 10 & 595.359 & 34.641 & 630 \\
\hline $45^{\circ}$ & 50 & 10 & 610 & 20 & 630 \\
\hline $50^{\circ}$ & 50 & 10 & 613.22 & 16.78 & 630 \\
\hline
\end{tabular}

\section{Tapered flatted end tube analyze in Ansys}

Tapered flatted end tube is exported to ANSYS in "igse format. In the design modular of ansys work bench, Steel material of Fe250 is assigned to the exported tapered flatted end tubes. When the tapered flatted end tubes are assigned with material property, there appears a green tick $(\sqrt{ })$ mark which indicates, that the model is ready for meshing. Parts are meshed individually and have no connections with each other. Automatic unstructured mesh generation is made for all parts. 
The load is applied in bolt fixed in vertical upward and downward direction in gradually incremental order. Load applied from $10 \mathrm{kN}$ to $50 \mathrm{kN}$ in an incremental order of $2 \mathrm{kN}$. When some external load is applied on the tube, the principal stresses and shear stresses are produced in the tube. According to theories of failure, if principal stresses and shear stress are produced in the tapered tube due to the application of the load, is the

beyond the allowable tensile stress and allowable compressive stress, the tube is said to have "failed".

\section{RESULT AND DISCUSSION}

Results of shear stress for tensile loading

For failure material

$$
\begin{gathered}
1 / 2(\sigma 1-\sigma 3) \geq 1 / 2 \sigma^{*} \mathrm{t} \\
\text { Or } \\
(\sigma 1-\sigma 3) \geq \sigma^{*} \mathrm{t}
\end{gathered}
$$

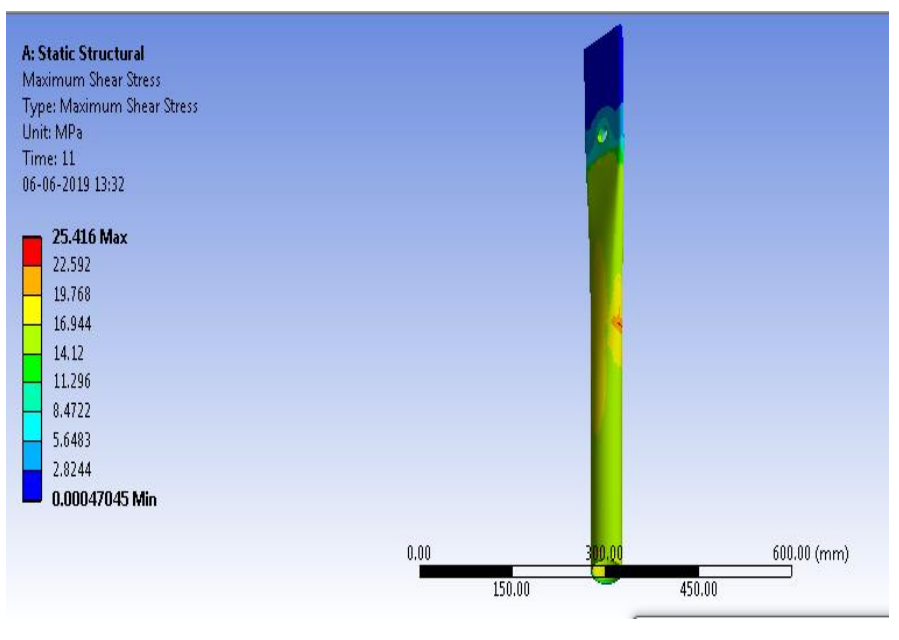

\section{$5^{\circ}$ Tapered flattened end tube}

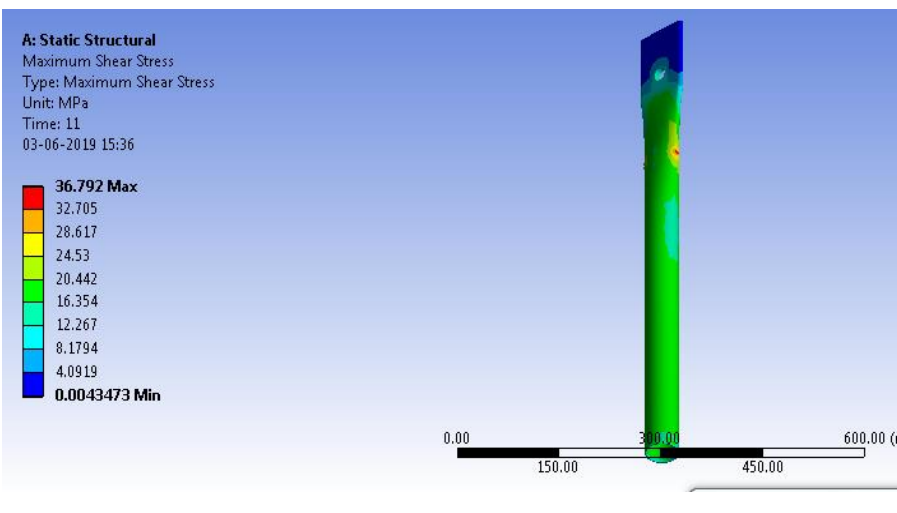

$10^{\circ}$ Tapered flattened end tube

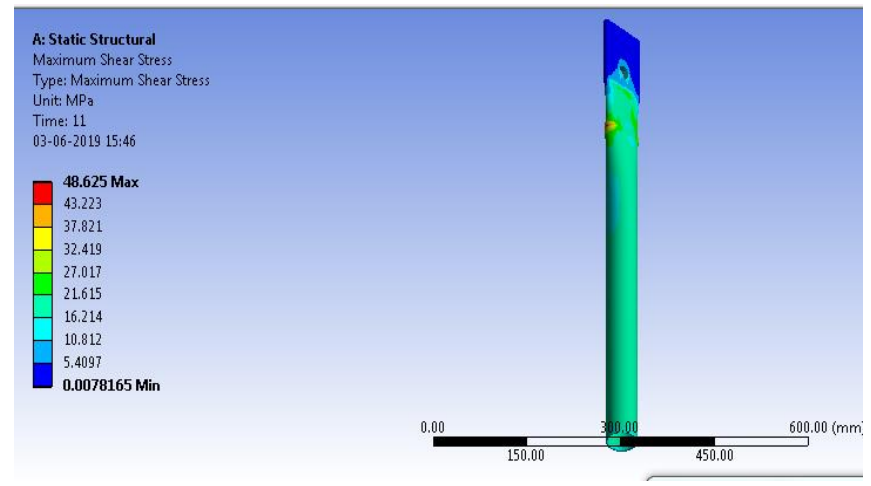

$15^{\circ}$ Tapered flattened end tube

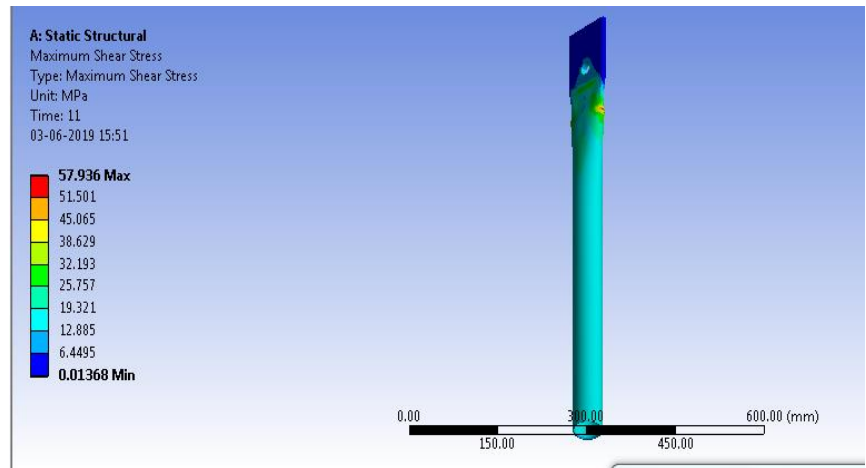

$20^{\circ}$ Tapered flattened end tube

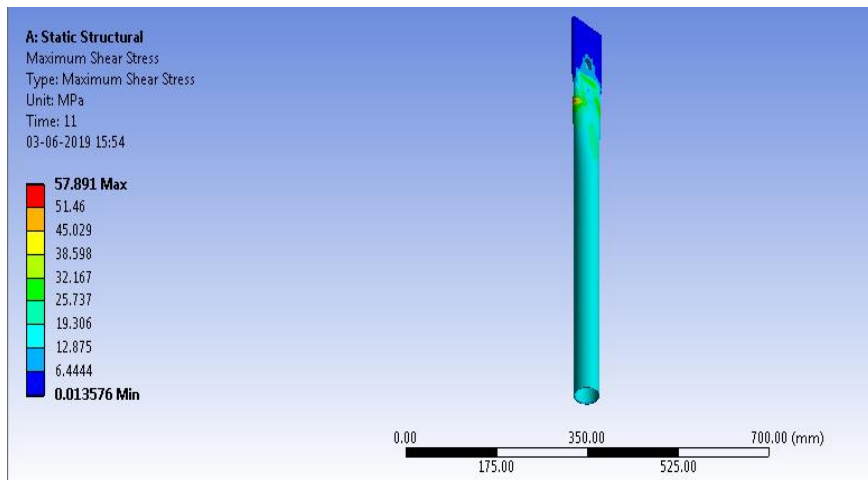

$30^{\circ}$ Tapered flattened end tube

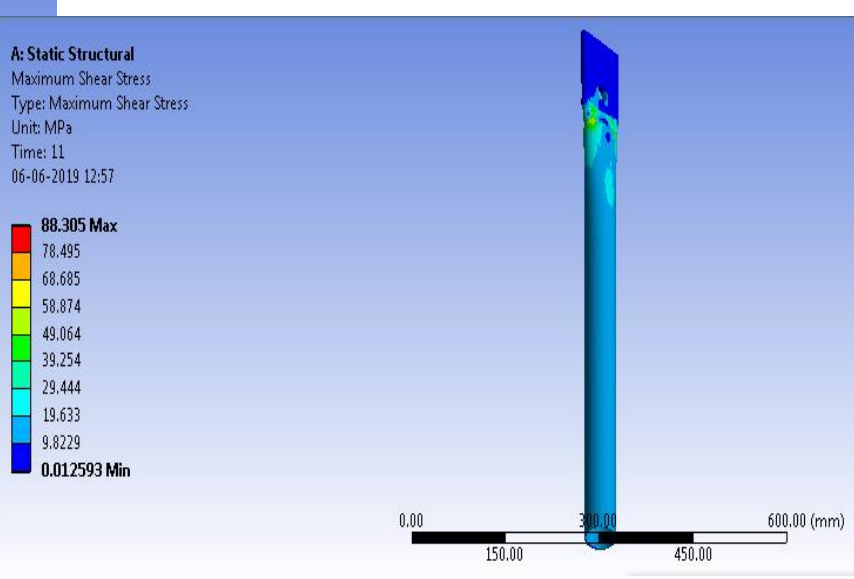

$45^{\circ}$ Tapered flattened end tube

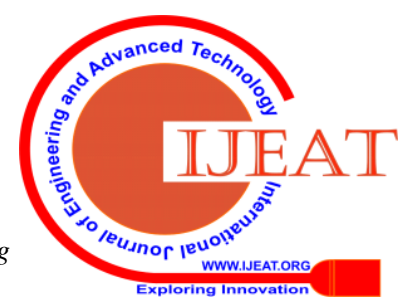




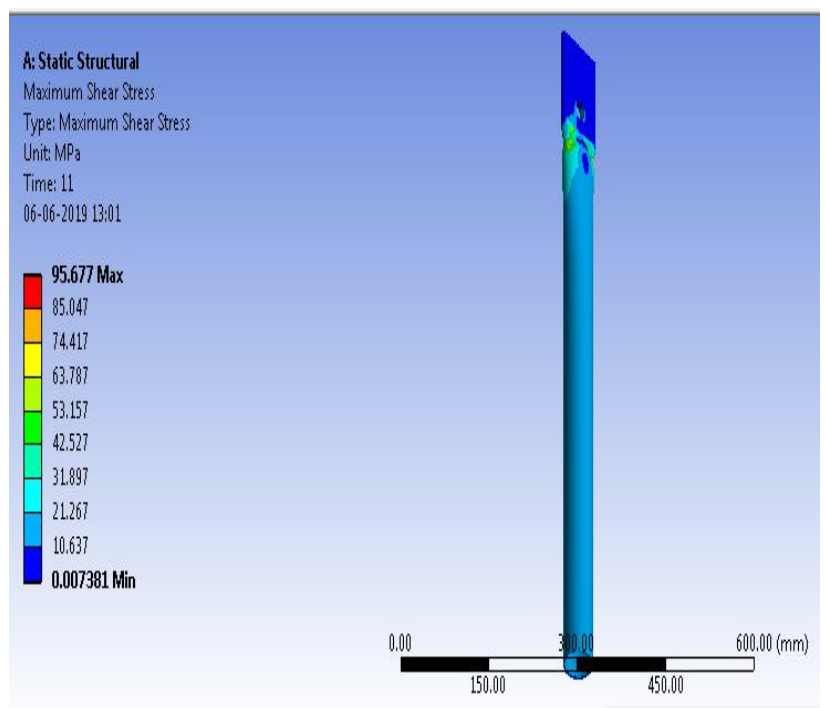

$50^{\circ}$ Tapered flattened end tube

Fig 3. Maximum Shear Stress For 10kn Tensile Load

\begin{tabular}{|c|c|c|c|c|c|c|c|c|c|c|}
\hline \multirow{2}{*}{ Angle } & \multicolumn{7}{|c|}{ Maximum Shear stress in N/.mm ${ }^{2}$} \\
\cline { 2 - 12 } & $\mathbf{5} \mathbf{~ k N}$ & $\mathbf{1 0 ~ k N}$ & $\mathbf{1 5 k N}$ & $\mathbf{2 0 ~ k N}$ & $\mathbf{2 5 ~ k N}$ & $\mathbf{3 0 ~ k N}$ & $\mathbf{3 5 k N}$ & $\mathbf{4 0 ~ k N}$ & $\mathbf{4 5} \mathbf{~ k N}$ & $\mathbf{5 0 ~ k N}$ \\
\hline $5^{\circ}$ & 12.70 & 25.41 & 38.12 & 50.83 & 63.53 & 76.24 & 88.955 & 101.66 & 114.37 & 127.08 \\
\hline $10^{\circ}$ & 18.39 & 36.79 & 55.18 & 73.58 & 91.98 & 110.38 & 128.77 & 147.17 & 165.56 & 183.96 \\
\hline $15^{\circ}$ & 24.31 & 48.62 & 72.93 & 97.25 & 121.56 & 145.87 & 170.19 & 194.5 & 218.81 & 243.12 \\
\hline $20^{\circ}$ & 28.96 & 57.93 & 86.90 & 115.87 & 144.84 & 173.81 & 202.78 & 231.75 & 260.71 & 289.68 \\
\hline $30^{\circ}$ & 28.94 & 57.89 & 86.83 & 115.78 & 144.73 & 173.67 & 202.62 & 231.56 & 260.51 & 289.45 \\
\hline $45^{\circ}$ & 44.15 & 88.30 & 132.4 & 176.61 & 220.76 & 264.92 & 309.07 & 353.22 & 397.37 & 441.53 \\
\hline $50^{\circ}$ & 47.83 & 95.67 & 143.5 & 191.35 & 239.19 & 287.03 & 334.87 & 382.71 & 430.55 & 478.38 \\
\hline
\end{tabular}

For design the following equation should be used

$$
1 / 2(\sigma 1-\sigma 3)=1 / 2 \sigma t
$$

$\sigma \mathrm{t}=$ Allowable simple tensile stress

$\sigma \mathrm{t}$ = Allowable simple tensile stress $=151.197$
Table 3: Design load for all tapered end tube

\begin{tabular}{|c|c|}
\hline $\begin{array}{c}\text { Flattening } \\
\text { Angle }\end{array}$ & $\begin{array}{c}\text { Design } \\
\text { load }(\mathrm{kN})\end{array}$ \\
\hline $\mathbf{5}^{\circ}$ & 48 \\
\hline $\mathbf{1 0}^{\circ}$ & 33.97 \\
\hline $\mathbf{1 5}^{\circ}$ & 25.65 \\
\hline $\mathbf{2 0}^{\circ}$ & 18.57 \\
\hline $\mathbf{3 0}^{\circ}$ & 18.55 \\
\hline $\mathbf{4 5}^{\circ}$ & 14.16 \\
\hline $\mathbf{5 0}^{\circ}$ & 13.08 \\
\hline
\end{tabular}

Results of Principal stress for tensile loading: 
Then according this theory, the failure will take place if $\sigma 1 \geq \sigma^{*} \mathrm{t}$ in simple tension

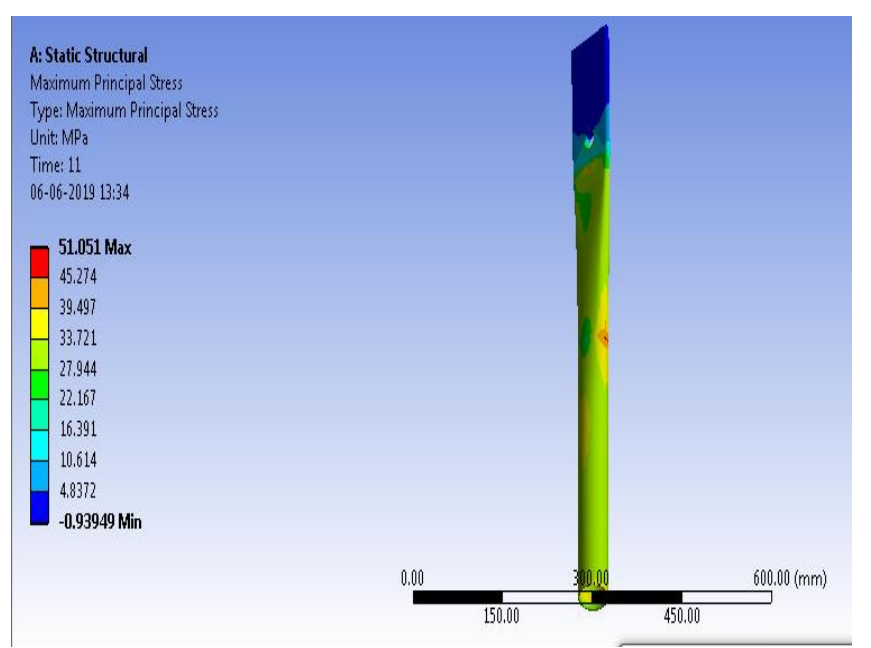

\section{$5^{\circ}$ Tapered flattened end tube}

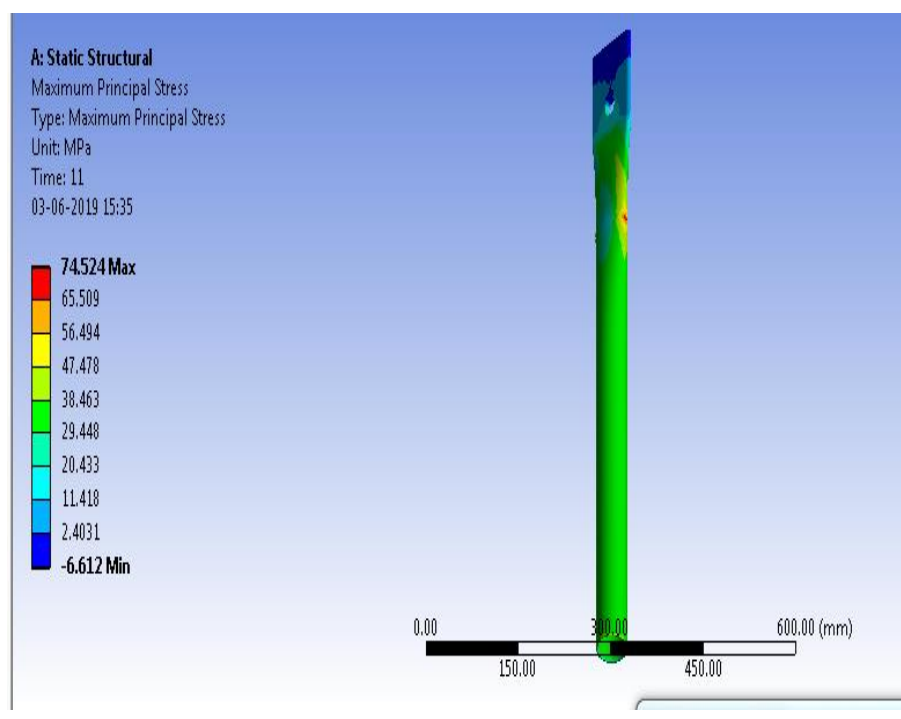

$10^{\circ}$ Tapered flattened end tube

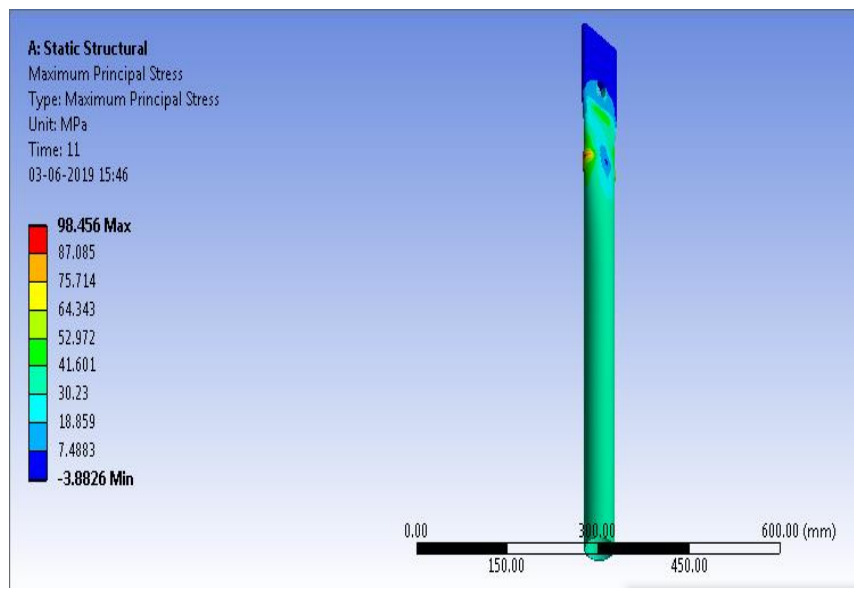

$15^{\circ}$ Tapered flattened end tube

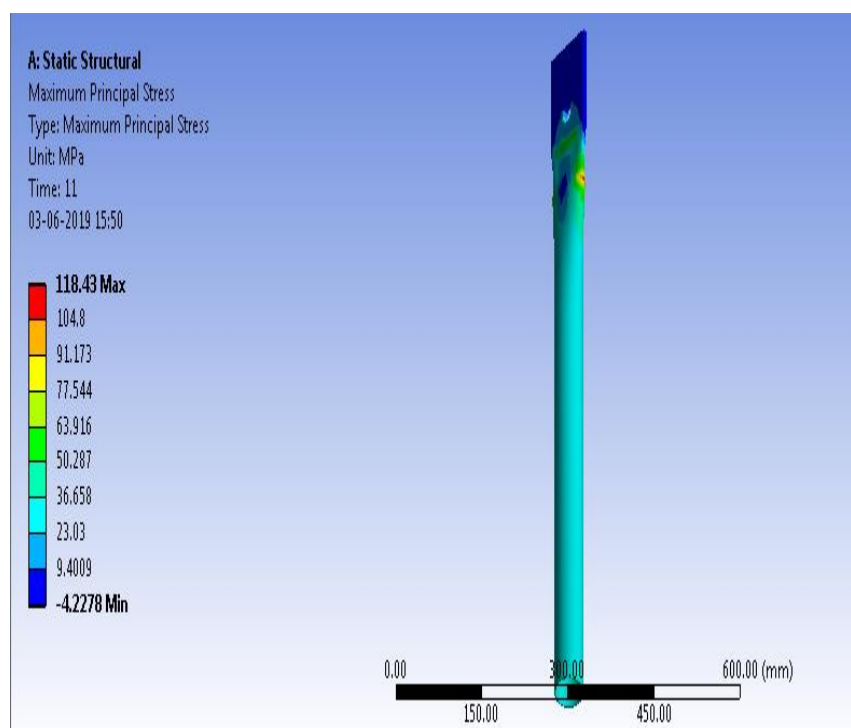

$20^{\circ}$ Tapered flattened end tube

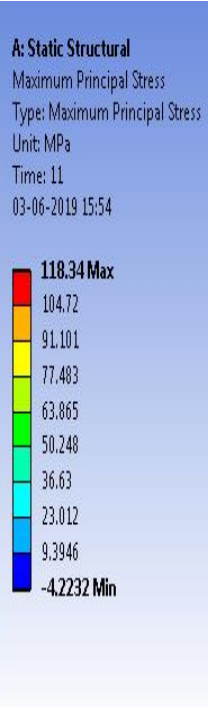

$30^{\circ}$ Tapered flattened end tube

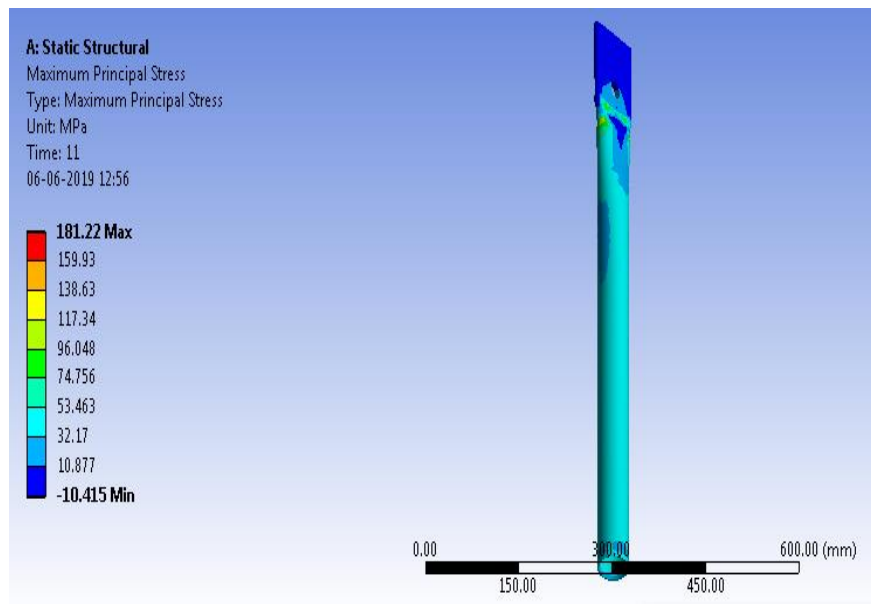

$45^{\circ}$ Tapered flattened end tube

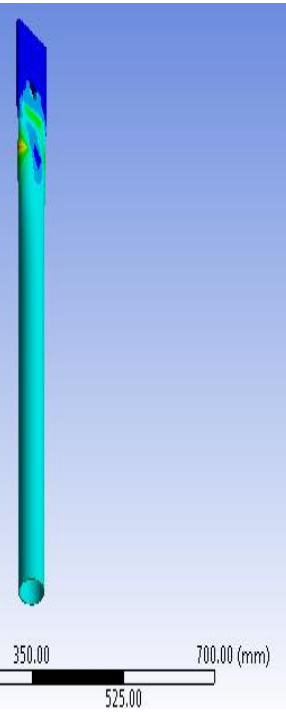

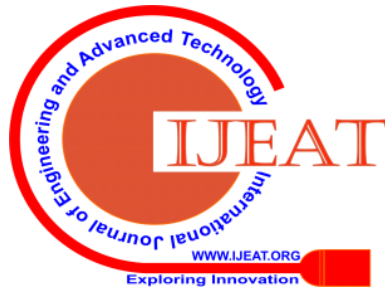




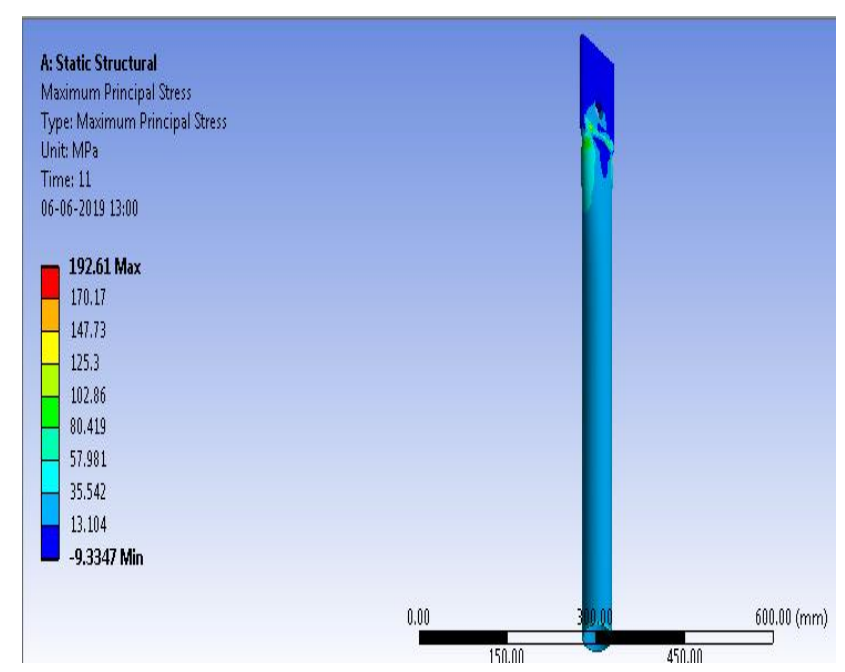

$50^{\circ}$ Tapered flattened end tube

Fig 4. Maximum Principal Stress for $10 \mathrm{kN}$ tensile load

\begin{tabular}{|c|c|c|c|c|c|c|c|c|c|c|}
\hline \multirow{2}{*}{ Angle } & \multicolumn{8}{|c|}{ Maximum Principal stress in N/mm } \\
\cline { 2 - 12 } & $\mathbf{5 ~ k N}$ & $\mathbf{1 0 ~ k N}$ & $\mathbf{1 5 k N}$ & $\mathbf{2 0 ~ k N}$ & $\mathbf{2 5} \mathbf{~ k N}$ & $\mathbf{3 0 ~ k N}$ & $\mathbf{3 5 k N}$ & $\mathbf{4 0 ~ k N}$ & $\mathbf{4 5} \mathbf{~ k N}$ & $\mathbf{5 0 ~ k N}$ \\
\hline $5^{\circ}$ & 25.525 & 51.051 & 76.57 & 102.1 & 127.6 & 153.15 & 178.68 & 204.2 & 229.73 & 255.25 \\
\hline $10^{\circ}$ & 37.262 & 74.524 & 111.7 & 149.0 & 186.3 & 223.5 & 260.83 & 298.09 & 335.36 & 372.62 \\
\hline $15^{\circ}$ & 49.228 & 98.456 & 147.6 & 196.9 & 246.1 & 295.37 & 344.6 & 393.82 & 443.05 & 492.28 \\
\hline $20^{\circ}$ & 59.215 & 118.43 & 177.6 & 236.8 & 296.0 & 355.29 & 414.51 & 473.72 & 532.94 & 592.15 \\
\hline $30^{\circ}$ & 59.168 & 118.34 & 177.5 & 236.6 & 295.8 & 355.01 & 414.18 & 473.35 & 532.51 & 591.68 \\
\hline $45^{\circ}$ & 90.61 & 181.22 & 271.8 & 362.4 & 453.0 & 543.66 & 634.27 & 724.88 & 815.49 & 906.1 \\
\hline $50^{\circ}$ & 96.306 & 192.61 & 288.9 & 385.2 & 481.5 & 577.84 & 674.14 & 770.45 & 866.75 & 963.06 \\
\hline
\end{tabular}

If maximum principal stress is the design criterion, and then maximum principal stress must not exceed the permissible stress $(\sigma t)$ for the given material.

\section{Hence $\sigma 1=\sigma \mathrm{t}$}

\section{Table 5: Design load for all tapered end tube}

\begin{tabular}{|c|c|}
\hline $\begin{array}{c}\text { Flattening } \\
\text { Angle }\end{array}$ & $\begin{array}{c}\text { Design } \\
\text { load } \\
(\mathrm{kN})\end{array}$ \\
\hline $5^{\circ}$ & 24.48 \\
\hline $10^{\circ}$ & 16.77 \\
\hline $15^{\circ}$ & 12.70 \\
\hline $20^{\circ}$ & 10.55 \\
\hline $30^{\circ}$ & 10.90 \\
\hline $45^{\circ}$ & 6.89 \\
\hline $50^{\circ}$ & 6.489 \\
\hline
\end{tabular}

Compression loading for tapered flattened end tube Results for Shear stress for compression loading

With tensile load, compression also to be checked. Maximum principal stresses for all models are tabulated in table. Allowable compression stress at elastic limit in simple compression stress as per IS $800-2007$ is $140.255 \mathrm{Mpa}$.

For failure material

$$
\begin{gathered}
1 / 2(\sigma 1-\sigma 3) \geq 1 / 2 \sigma^{*} c \\
\text { Or } \\
(\sigma 1-\sigma 3) \geq \sigma^{*} c
\end{gathered}
$$




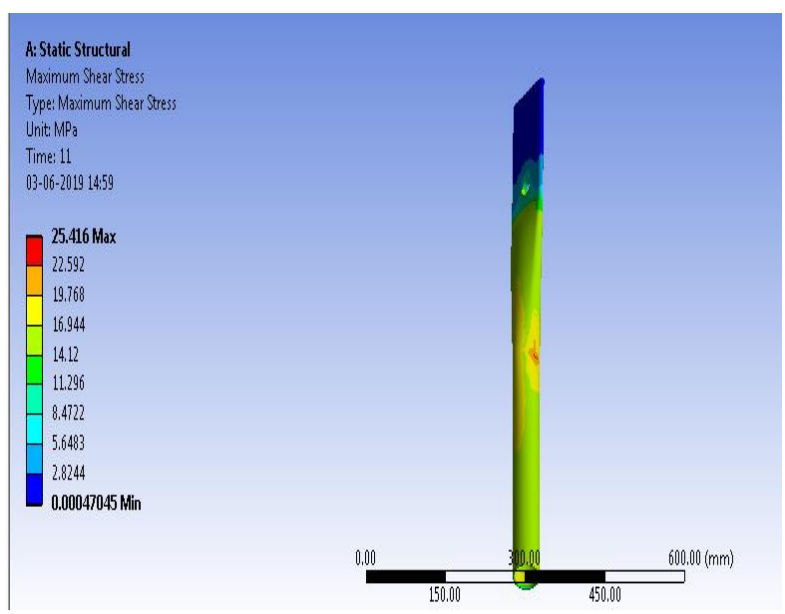

$5^{\circ}$ Tapered flattened end tube

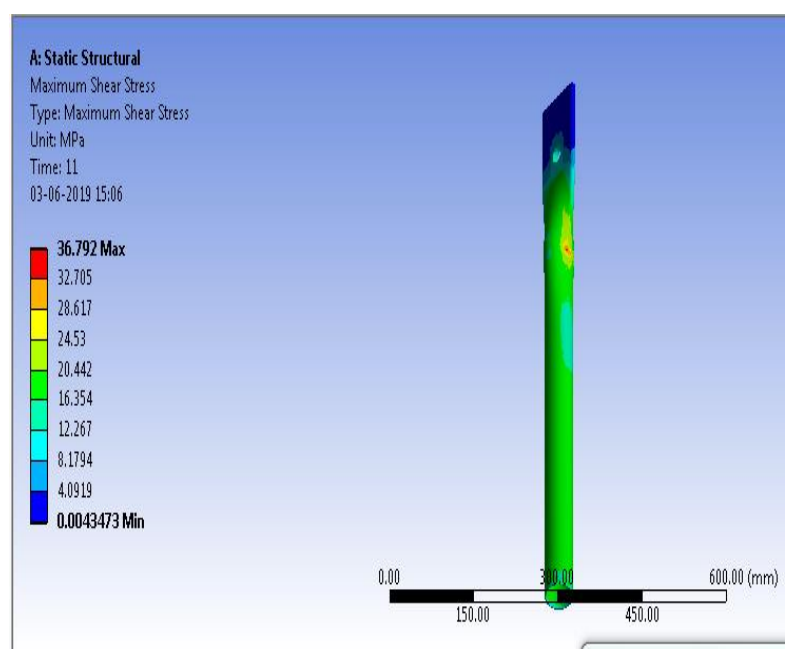

$10^{\circ}$ Tapered flattened end tube

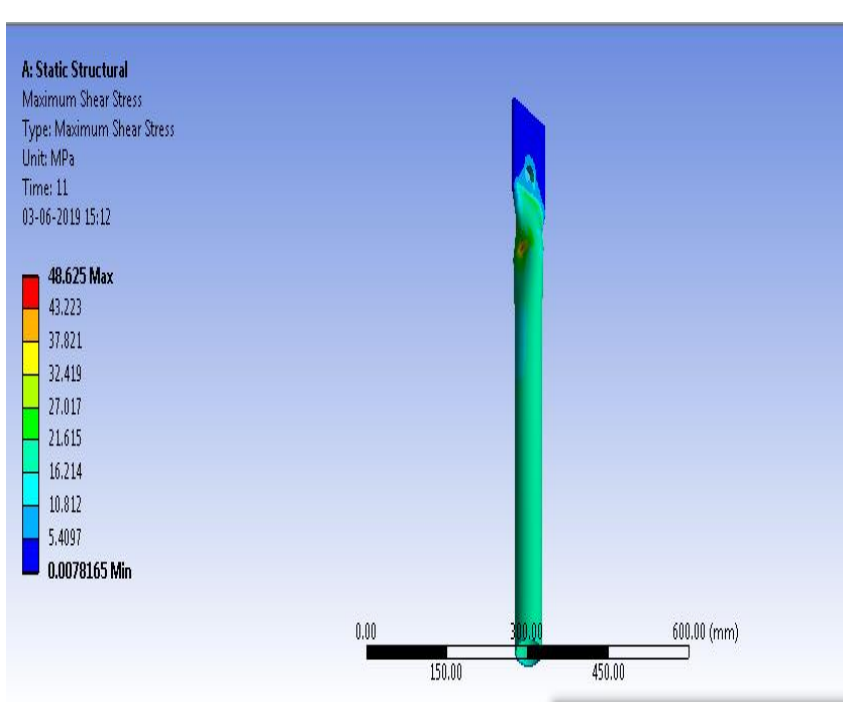

$15^{\circ}$ Tapered flattened end tube

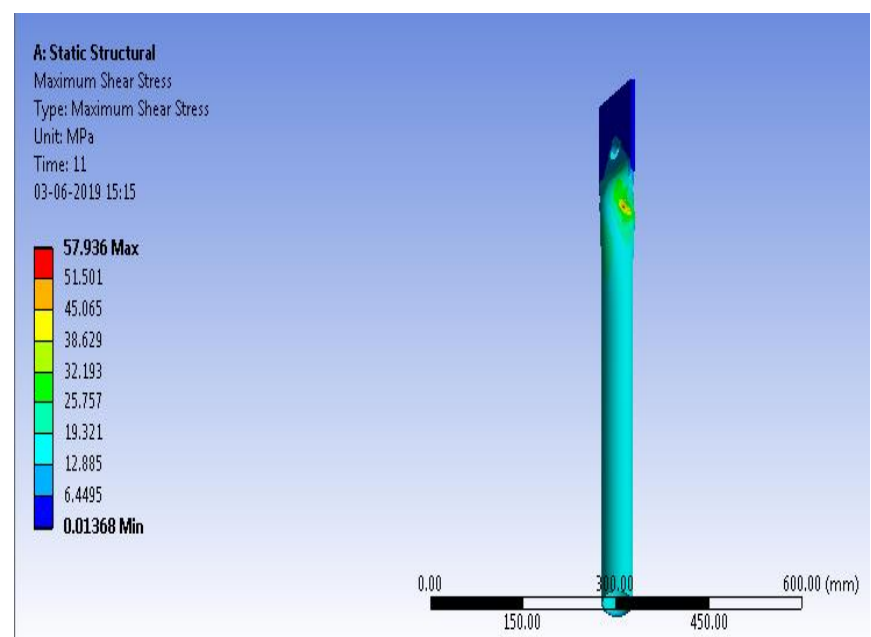

$20^{\circ}$ Tapered flattened end tube

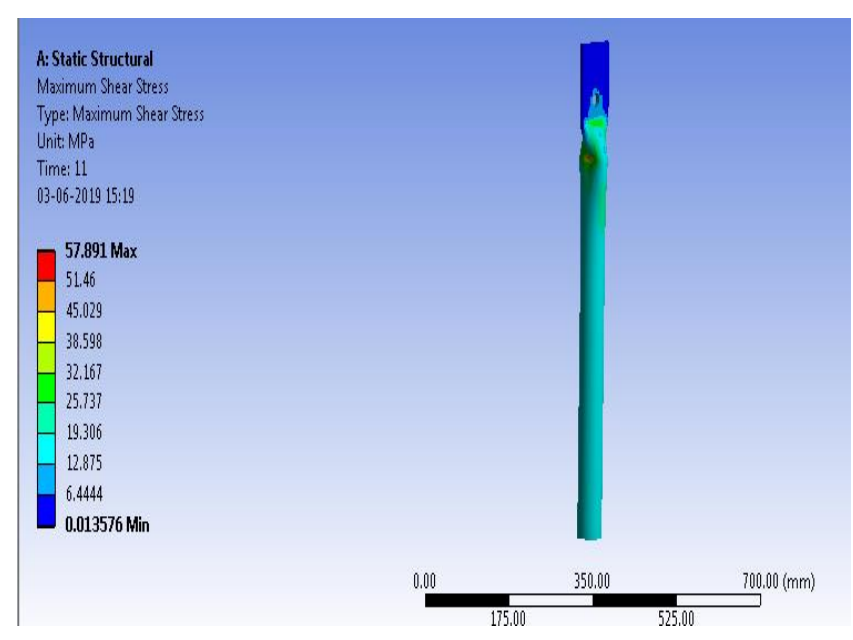

$30^{\circ}$ Tapered flattened end tube

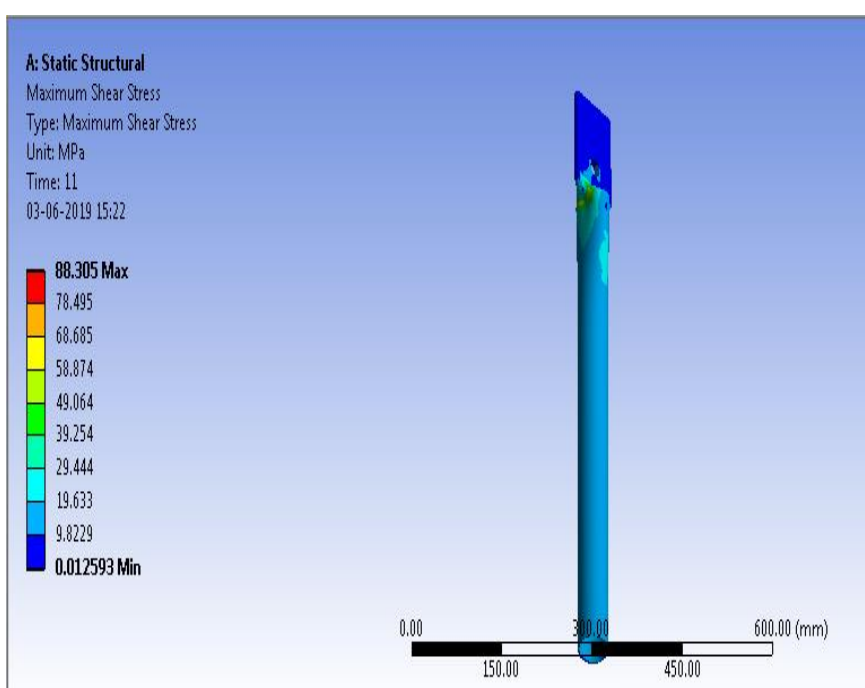

$45^{\circ}$ Tapered flattened end tube

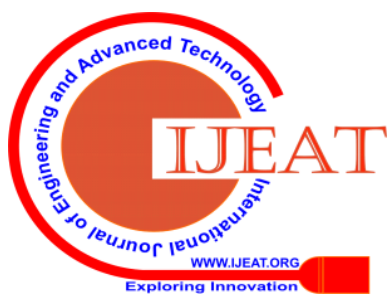




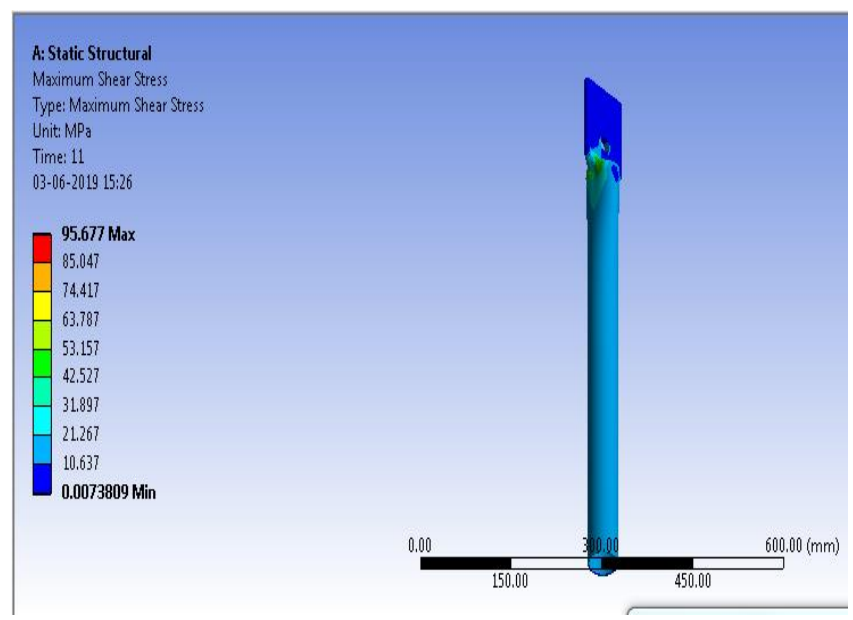

$50^{\circ}$ Tapered flattened end tube

Fig 5. Maximum Shear Stress for $10 \mathrm{kN}$ compression load

\begin{tabular}{|c|c|c|c|c|c|c|c|c|c|c|}
\hline \multirow{2}{*}{ Angle } & \multicolumn{10}{|c|}{ Maximum Principal stress in $\mathbf{N} / \mathrm{mm}^{2}$} \\
\hline & $5 \mathrm{kN}$ & $10 \mathrm{kN}$ & $15 k \mathbf{k}$ & $20 \mathrm{kN}$ & $25 \mathrm{kN}$ & $30 \mathbf{k N}$ & $35 \mathbf{k N}$ & 40 kN & $45 \mathrm{kN}$ & $50 \mathrm{kN}$ \\
\hline $5^{\circ}$ & 12.708 & 25.416 & 38.12 & 50.83 & 63.539 & 76.247 & 88.955 & 101.66 & 114.37 & 127.08 \\
\hline $10^{\circ}$ & 18.396 & 36.792 & 55.188 & 73.584 & 91.98 & 110.38 & 128.77 & 147.17 & 165.56 & 183.96 \\
\hline $15^{\circ}$ & 24.312 & 48.625 & 72.937 & 97.25 & 121.56 & 145.87 & 170.19 & 194.5 & 218.81 & 243.12 \\
\hline $20^{\circ}$ & 28.968 & 57.936 & 86.905 & 115.87 & 144.84 & 173.81 & 202.78 & 231.75 & 260.71 & 289.68 \\
\hline $30^{\circ}$ & 28.945 & 57.891 & 86.836 & 115.78 & 144.73 & 173.67 & 202.62 & 231.56 & 260.51 & 289.45 \\
\hline $45^{\circ}$ & 44.153 & 88.305 & 132.46 & 176.61 & 220.76 & 264.92 & 309.07 & 353.22 & 397.37 & 441.53 \\
\hline $50^{\circ}$ & 47.838 & 95.677 & 143.52 & 191.35 & 239.19 & 287.03 & 334.87 & 382.71 & 430.55 & 478.38 \\
\hline
\end{tabular}

For design the following equation should be used

$$
1 / 2(\sigma 1-\sigma 3)=1 / 2 \sigma c
$$

$\sigma \mathrm{c}=$ Allowable simple compressive stress

$$
\sigma \mathrm{c}=140.255 \mathrm{Mpa}
$$

Table 7: Design load for all flattening angle

\begin{tabular}{|c|c|}
\hline $\begin{array}{c}\text { Flattening } \\
\text { Angle }\end{array}$ & $\begin{array}{c}\text { Design load } \\
(\mathrm{kN})\end{array}$ \\
\hline $\mathbf{5}^{\circ}$ & 27.6 \\
\hline $\mathbf{1 0}^{\circ}$ & 19.06 \\
\hline $\mathbf{1 5}^{\circ}$ & 14.422 \\
\hline $\mathbf{2 0}^{\circ}$ & 12.104 \\
\hline $\mathbf{3 0}^{\circ}$ & 12.11 \\
\hline $\mathbf{4 5}^{\circ}$ & 7.941 \\
\hline $\mathbf{5 0}^{\circ}$ & 7.33 \\
\hline
\end{tabular}

Results for Maximum Principal stress for compression loading

Then according this theory, the failure will take place if $|\sigma 3| \geq \sigma^{*} \mathrm{c}$ in simple compression

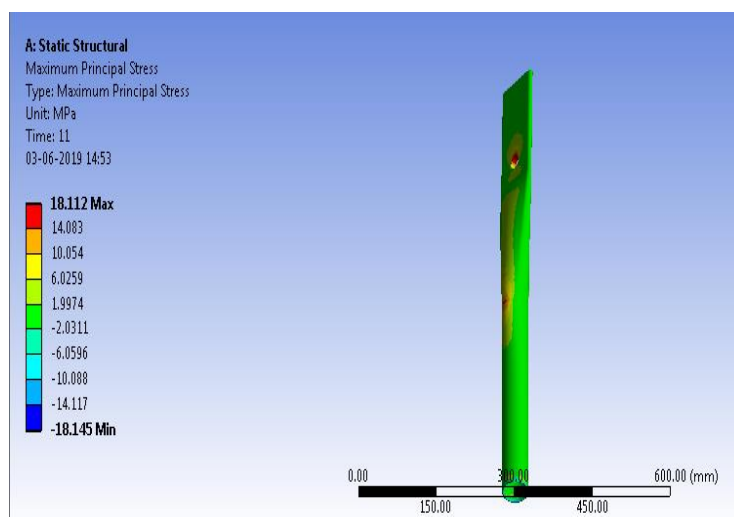

$5^{\circ}$ Tapered flattened end tube 


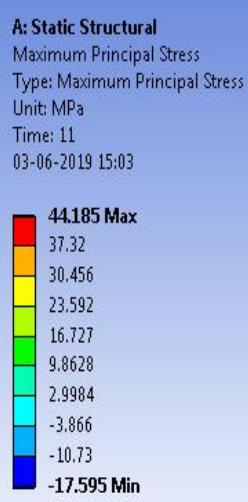

-17.595 Min

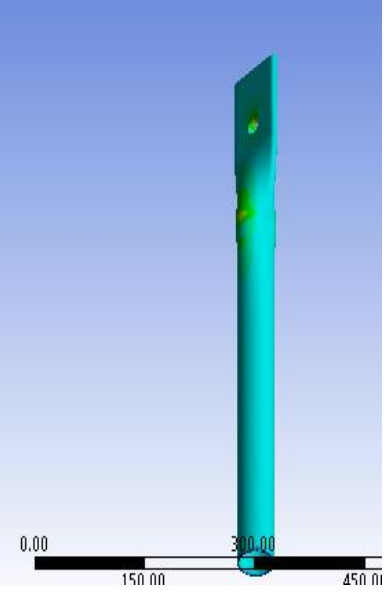

$10^{\circ}$ Tapered flattened end tube

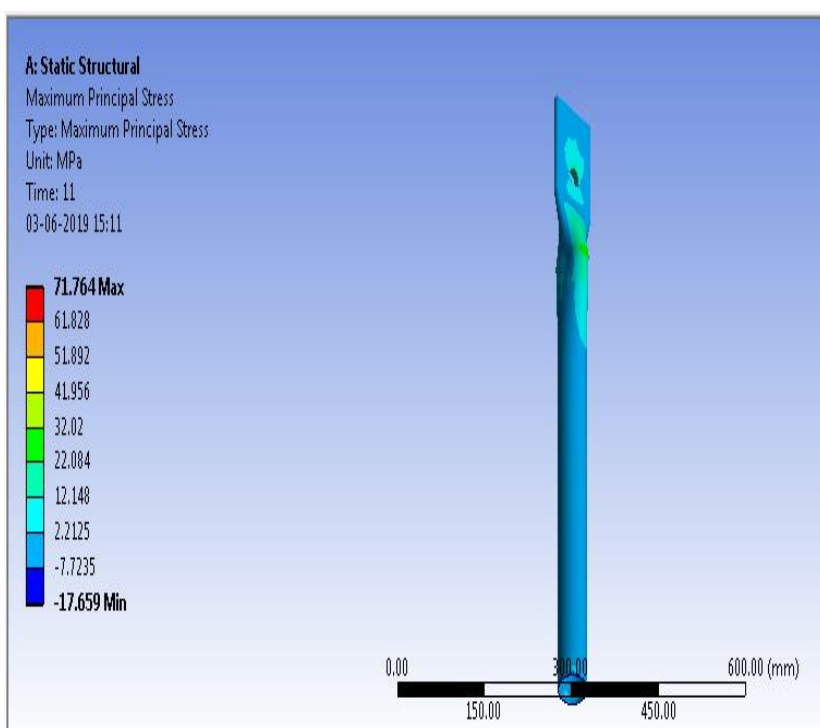

$15^{\circ}$ Tapered flattened end tube

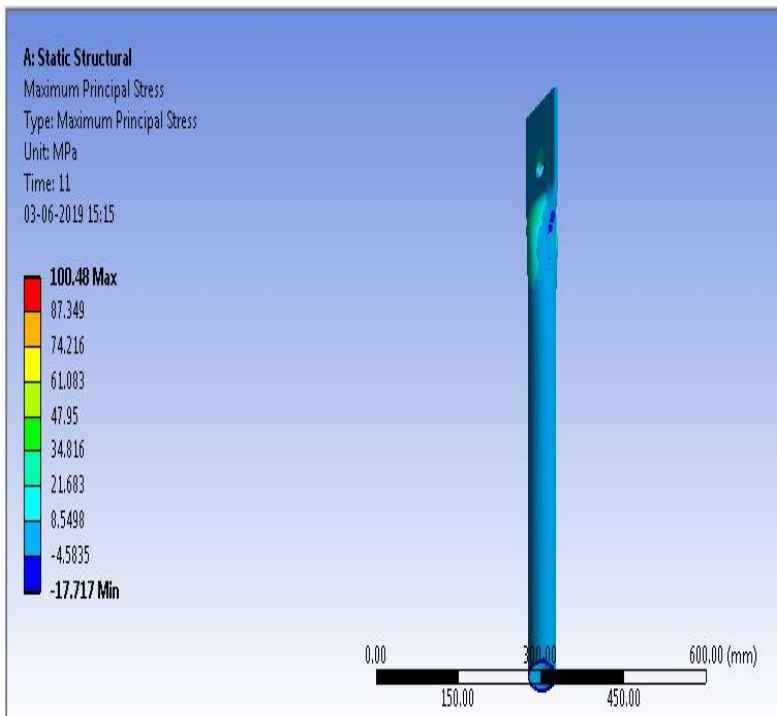

$20^{\circ}$ Tapered flattened end tube

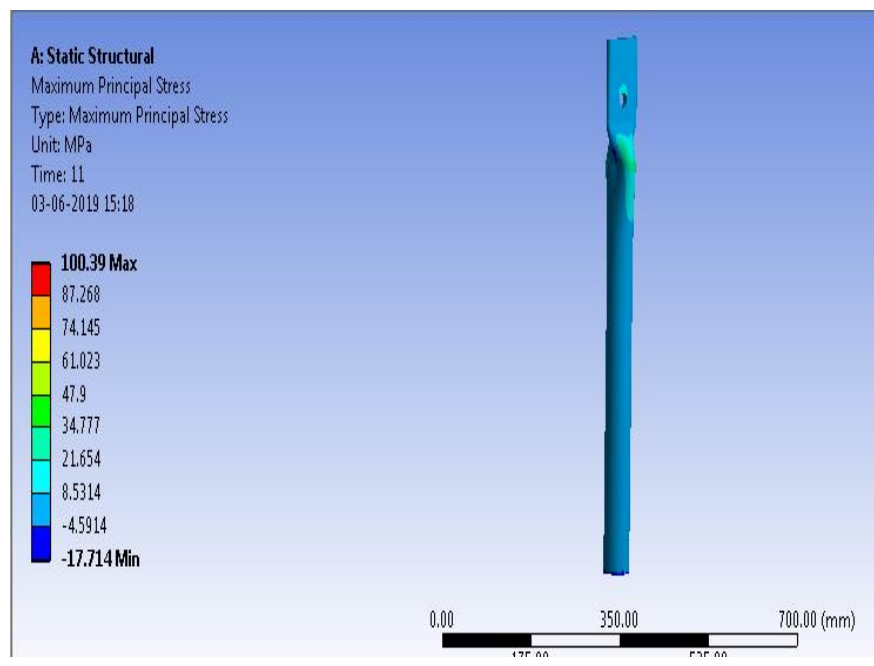

$30^{\circ}$ Tapered flattened end tube

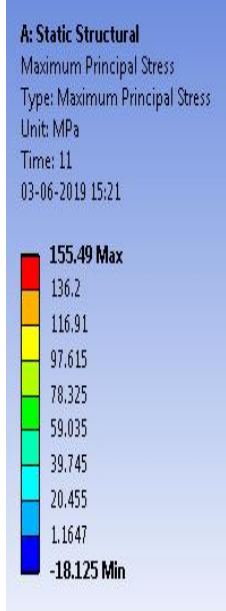

$45^{\circ}$ Tapered flattened end tube

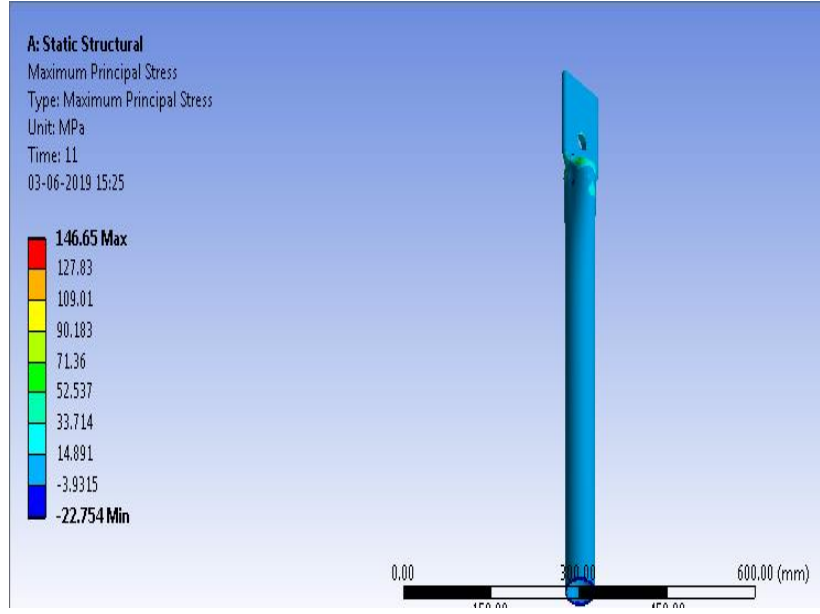

$50^{\circ}$ Tapered flattened end tube

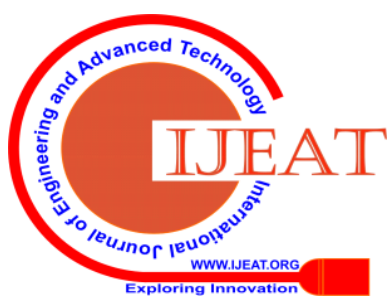


Design of Tapered Flatted End Tube for Tension and Compression Loading

Fig 6. Maximum principal Stress for $10 \mathrm{kN}$ compression load

III. CONCLUSION

1) As per the maximum shear stress theories criteria for

\begin{tabular}{|c|c|c|c|c|c|c|c|c|c|c|}
\hline \multirow{2}{*}{ Angle } & \multicolumn{10}{|c|}{ Maximum Principal stress in $\mathbf{N} / \mathrm{mm}^{2}$} \\
\hline & $5 \mathbf{k N}$ & $10 \mathbf{k N}$ & $15 \mathrm{kN}$ & $20 \mathrm{kN}$ & $25 \mathbf{k N}$ & $30 \mathbf{~ k N}$ & $35 \mathbf{k N}$ & $40 \mathrm{kN}$ & $45 \mathrm{kN}$ & $50 \mathrm{kN}$ \\
\hline $5^{\circ}$ & 9.0558 & 18.112 & 27.167 & 36.223 & 45.279 & 54.335 & 63.39 & 72.446 & 81.502 & 90.558 \\
\hline $10^{\circ}$ & 22.092 & 44.185 & 66.277 & 88.369 & 110.46 & 132.55 & 154.65 & 176.74 & 198.83 & 220.92 \\
\hline $15^{\circ}$ & 35.882 & 71.764 & 107.65 & 143.53 & 179.41 & 215.29 & 251.18 & 287.06 & 322.94 & 358.82 \\
\hline $20^{\circ}$ & 59.215 & 118.43 & 177.65 & 236.86 & 296.08 & 355.29 & 414.51 & 473.72 & 532.94 & 592.15 \\
\hline $30^{\circ}$ & 50.195 & 100.39 & 150.59 & 200.78 & 250.98 & 301.17 & 351.37 & 401.56 & 451.76 & 501.95 \\
\hline $45^{\circ}$ & 77.743 & 155.49 & 233.23 & 310.97 & 388.71 & 466.46 & 544.2 & 621.94 & 699.69 & 777.43 \\
\hline $50^{\circ}$ & 73.326 & 146.65 & 219.98 & 293.3 & 366.63 & 439.95 & 513.28 & 586.6 & 659.93 & 733.26 \\
\hline
\end{tabular}

If the maximum principal stress is the design criterion, then maximum principal stress must not exceed the permissible stress $(\sigma c)$ for the given material.

Hence $\sigma 3=\sigma c$

Table 9: Design load for all tapered end tube

\begin{tabular}{|c|c|}
\hline $\begin{array}{c}\text { Flattening } \\
\text { Angle }\end{array}$ & $\begin{array}{c}\text { Design load } \\
(\mathrm{KN})\end{array}$ \\
\hline $\mathbf{5}^{\circ}$ & $\begin{array}{c}\text { More than } \\
50 \mathrm{kN}\end{array}$ \\
\hline $\mathbf{1 0}^{\circ}$ & 31.743 \\
\hline $\mathbf{1 5}^{\circ}$ & 19.57 \\
\hline $\mathbf{2 0}^{\circ}$ & 11.831 \\
\hline $\mathbf{3 0}^{\circ}$ & 13.98 \\
\hline $\mathbf{4 5}^{\circ}$ & 9.02 \\
\hline $\mathbf{5 0}^{\circ}$ & 9.56 \\
\hline
\end{tabular}

tension loading the least design load is in the $50^{\circ}$ model. The effectiveness in reducing design load is in the order of $5^{\circ}$, $10^{\circ}, 15,30^{\circ} \circ, 20^{\circ}$, and $45^{\circ}$.

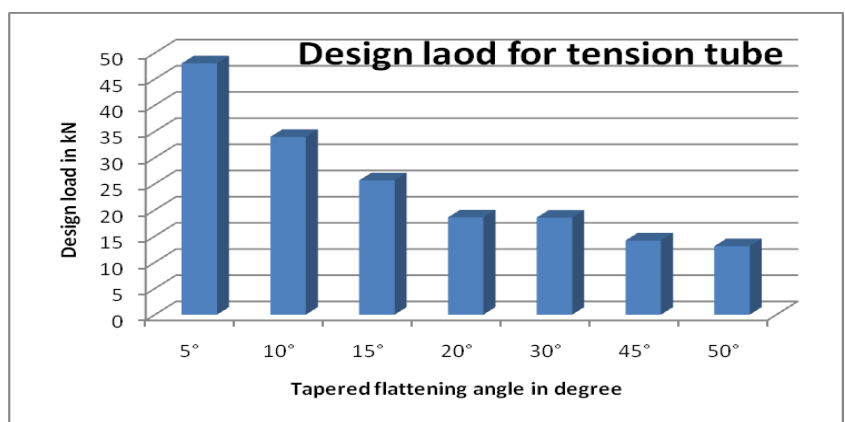

2) As per maximum principal stress theories for tension loading the least design load is in the $50^{\circ}$ model. The effectiveness in reducing design load is in the order of $5^{\circ}$, $10^{\circ}, 15^{\circ}, 30^{\circ}, 20^{\circ}$ and $45^{\circ}$.

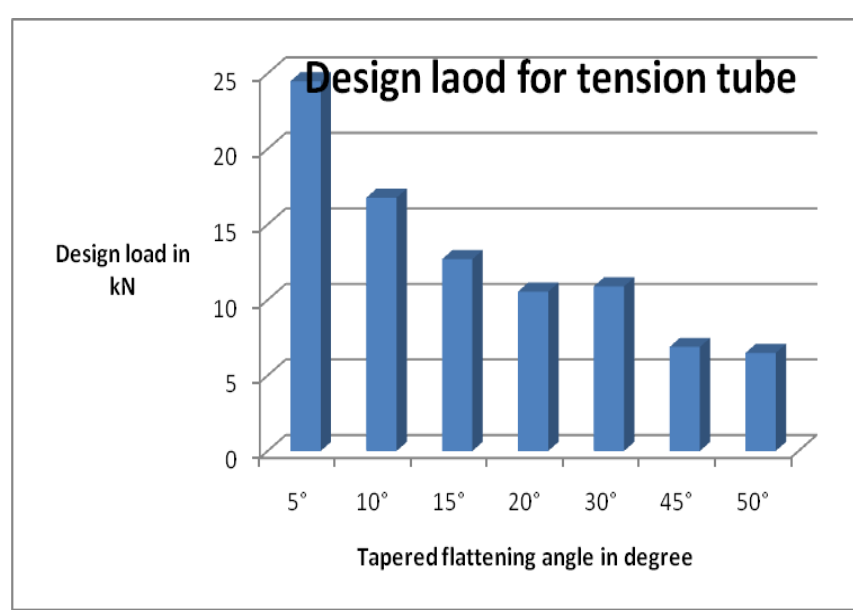


3) As per maximum shear stress theories for compression loading the least design load is in the $50^{\circ}$ model. The effectiveness in reducing design load is in the order of $5^{\circ}$, $10^{\circ}, 15^{\circ}, 30^{\circ}, 20^{\circ}$ and $45^{\circ}$.

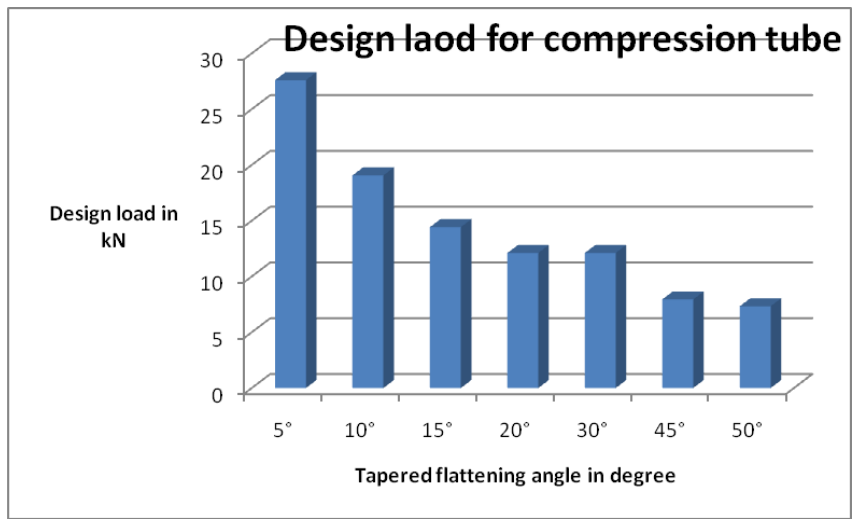

4) As per maximum Principal stress theories for compression loading the least design load is in the $45^{\circ}$ model. The effectiveness in reducing design load is in the order of $5^{\circ}$, $10^{\circ}, 15^{\circ}, 30^{\circ}, 20^{\circ}$ and $50^{\circ}$.

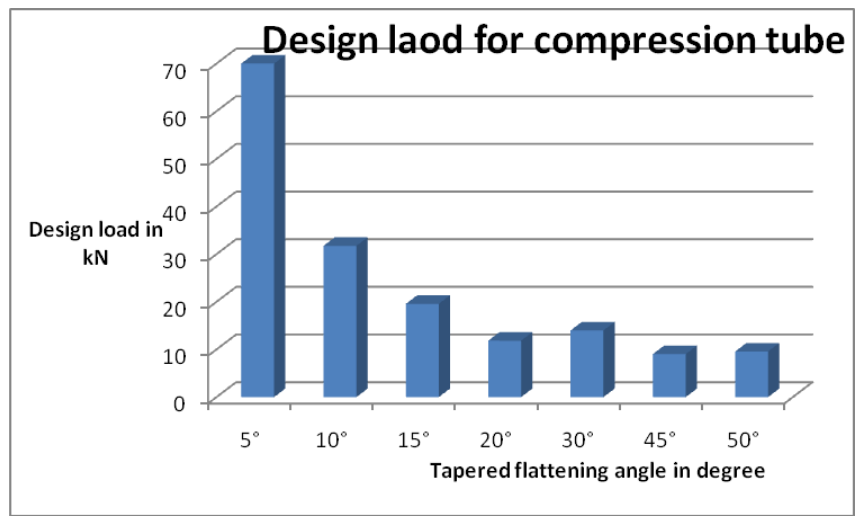

\section{REFERENCES}

1. I P Sonar and V R siddaye (2009), "theoretical and experimental investigation on single and double bolted bamboo joint under axial tension" Journal of structural engineering, Volume 36.No 3, Aug-spec. Year 2009.

2. NPTEL "Design of Steel Structures" "Space structures - Principals and practice by Subramanian Narayanan"

3. Hassan K. Al Nageim \& T.J "Steel Structures Practical Design Studies".

4. N Subramanian "Steel Structures Design and Practice" 